\title{
Burnout, clima de segurança e condições de trabalho em profissionais hospitalares
}

\author{
Burnout, safety climate, and working conditions for hospital professionals \\ Burnout, clima de seguridad y condiciones de trabajo en profesionales de la \\ salud en hospitales
}

\author{
Alexsandro Luiz DE ANDRADE ${ }^{1, a}$ \\ Thiago Drumond MORAES ${ }^{a}$ \\ Antônio Marcos TOSOLI ${ }^{\mathrm{b}}$ \\ João WACHELKE \\ Universidade Federal do Espírito Santo, Vitória, ES, Brasila ${ }^{2}$, Universidade do Estado do Rio de Janeiro, Rio de \\ Janeiro, RJ, Brasil ${ }^{b}$, Universidade Federal de Uberlândia, Uberlândia, MG, Brasil ${ }^{c}$
}

Resumo Esta pesquisa apresenta resultados de um modelo teórico-empírico que articula aspectos do contexto de trabalho, do clima de segurança e das variáveis psicossociais do trabalho em contexto hospitalar e suas decorrências na manifestação da síndrome do burnout. O burnout é um transtorno relacionado ao trabalho e ao estresse ocupacional severo, caracteriza-se pelo esgotamento emocional dos trabalhadores e é estabelecido por meio de determinadas associações entre características individuais, ambientais e laborais. Neste estudo objetivouse desenvolver e testar um modelo teórico-estrutural relacionando variáveis dimensionais de trabalho, burnout e saúde mental. A pesquisa teve um delineamento do tipo survey, cuja amostra, com caráter de conveniência, contou com 200 profissionais da área de saúde, sendo 174 participantes do sexo feminino (87\%), com média de idade de 34,4 anos e média de 6,5 anos de trabalho na profissão. O instrumento de pesquisa foi composto por escalas psicológicas dos seguintes construtos: a) clima de segurança no trabalho, b) contexto de trabalho, c) burnout e; d) aspectos sócio-demográficos. Os resultados do estudo indicaram relações existentes entre diferentes aspectos do trabalho, clima de segurança e saúde a partir de dois modelos teóricos integrativos de variáveis psicossociais, clima de segurança e saúde para trabalhadores em contexto hospitalar. Estes dados contribuem para orientar as políticas de promoção e proteção à saúde dos profissionais como estratégia central para garantir o serviço de prestação de cuidado na rotina diária do ambiente hospitalar.

Palavras-chave:

Burnout; hospital; condições de trabalho.

\section{Abstract}

This study presents results from a theoretical- empirical model that assembles aspects of the work context, safety climate, and psychosocial work variables in a hospital setting, and their consequences in the manifestation of burnout syndrome. Burnout is a disorder related to work associated with severe occupational stress. The syndrome is characterized by emotional exhaustion of workers and is established by means of certain associations among individual, environmental, and work characteristics. The goal of this study is to develop and test a structural theoretical model linking dimensional variables of work, burnout, and mental health. This is a survey type of design with a convenience sample of 200 health professionals, 174 of whom were female (87\%), mean age was 34.4 years, and with a mean of 6.5 years of work in the profession. The instrument for this research was composed of psychological scales for the following constructs: a) work security climate, b) work conditions, c) burnout, and d) socio-demographic aspects. The results indicate the existence of relationships between different aspects of work, safety climate, and health as observed through two integrative theoretical models of psychosocial variables, safety climate, and health for workers in hospital environments. These data help guide policies for worker health protection and prevention taken as a core strategy to ensure the provision of care within the daily routine of the hospital environment.

Keywords:

Burnout; hospital; work conditions.

Endereço para correspondência: Av. Fernando Ferrari, $n^{\circ}$ 514, Campus Universitário de Goiabeiras/UFES-CEMUNI VI, Vitória, ES, Brasil. 29075-910. E-mail: alexsandro.deandrade@yahoo.com 
Esta investigación presenta los resultados de un modelo teórico y empírico que articula aspectos del contexto de trabajo, del clima de seguridad y de las variables psicosociales del trabajo en un contexto hospitalario y sus consecuencias en la manifestación del síndrome de burnout. E1 Burnout es un trastorno relacionado con el trabajo asociado a lo estrés laboral grave. Este síndrome se caracteriza por el agotamiento emocional de los empleados y se establece por ciertas asociaciones entre las características individuales, ambientales y laborales. El objetivo de este estudio fue desarrollar y poner a prueba un modelo teórico-estructural relacionando variables dimensionales de trabajo, burnout y salud mental. Esta es una investigación con base en cuestionarios, cuya muestra, de carácter de conveniencia, contó con 200 profesionales de la salud, siendo 174 participantes del sexo femenino (87\%), con edad media 34,4 años y un promedio de 6,5 años de carrera. El instrumento consistió en escalas psicológicas de los siguientes constructos: a) clima de seguridad, b) contexto de trabajo, c) burnout y d) aspectos socio-demográficos. Los resultados del estudio indicaron que existe una relación entre los diferentes aspectos del trabajo, la salud y el clima de seguridad a partir de dos modelos teóricos que integran las variables psicosociales, el clima de seguridad y salud para los trabajadores en un contexto hospitalario. Los datos actuales contribuyen a orientar las políticas para promover y proteger a salud de los profesionales como estrategia central para asegurar el cuidado de servicio en la rutina diaria del ambiente hospitalario. Palabras-clave:

Burnout; hospital; condiciones de trabajo.

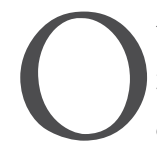

trabalho desenvolvido pelos profissionais da saúde no contexto hospitalar se apresenta como um fenômeno altamente complexo e de interesse antigo entre diversos pesquisadores (Bataile, 2014; Kovaleski \& Bressan, 2012; Pitta, 1990). Um dos textos clássicos da área da saúde mental do trabalhador (Pitta, 1990), apesar de não abordar o burnout diretamente, expõe os fatores psicossociais de risco do contexto institucional hospitalar que se caracteriza como propício à geração de estresse, desgaste emocional, fadiga extrema e despersonalização. A exposição às situações de sofrimento, à sensação frequente de impotência diante de quadros variados de dor, ao processo de morte e de morrer de pessoas de diferentes idades e em condições múltiplas, à possibilidade de adoecimento por doenças graves no curso do processo laboral e à constatação pessoal da finitude humana gera, em seu conjunto, uma estrutura social, física, emocional e psíquica que favorece o desgaste humano de forma intensa (Bataile, 2014; Kovaleski \& Bressan, 2012).

Os interesses de pesquisas no tema mantêm-se, ao longo do tempo, em função das características peculiares e dos desafios emocionais, psicológicos, físicos, mentais e orgânicos que estão implicados na realização diária desse trabalho. Acrescenta-se aos fatos anteriores as longas jornadas de trabalho, que variam entre 8 e 24 horas sucessivas. Considerando-se que é comum, ao menos em alguns estados do Brasil, o acúmulo de empregos tanto na rede básica quanto nas unidades hospitalares (Fernandes, Miranzi, Iwamoto, Tavares, \& Santos, 2012; Silva \& Melo, 2006), pode-se compreender a ocorrência de um processo de sobreposição entre os turnos de trabalho, que leva os profissionais a um estado de vigilância e tensão por muitas horas e, às vezes, dias seguidos (Nogueira-Martins, 2003). Em outro conjunto de questões sobre o trabalho em contexto hospitalar, encontra-se uma díade ligada à gestão e à formação de recursos humanos, que é a falta de profissionais para a demanda enfrentada no dia a dia do trabalho e a capacitação para a realização das ações e implementações dos serviços em saúde (Nicolau, 2014). É nesse complexo contexto profissional que emergem sintomas de adoecimento profissional, entre os quais destaca-se o burnout como um dos transtornos frequentemente investigados.

\section{Burnout e o esgotamento emocional no trabalho}

O burnout é um transtorno relacionado ao trabalho associado ao estresse ocupacional severo (Tamayo \& Tróccoli, 2009; Trigo, Teng, \& Hallak, 2007). Caracteriza-se como um transtorno relacionado a atividades de prestação de serviço em interação com pessoas (Murofuse, Abranches, \& Napoleão, 2005), razão pela qual vem sendo estudado com frequência em profissionais da saúde (Heeb \& Haberey-Knuessi, 2014) e da educação (Andrade \& Cardoso, 2012), embora haja estudos com trabalhadores das áreas mais diversas, como do setor bancário e da indústria de exploração florestal (Ahola, Salminen, Toppinen-Tanner, Koskinen, \& Vaananen, 2014; Amigo, Asensio, Menendez, Redondo, \& Ledesma, 2014).

Esse transtorno se caracteriza pelo esgotamento emocional dos trabalhadores e é estabelecido por meio de determinadas associações entre características individuais, ambientais e do trabalho (Trigo et al., 2007), particularmente quando há discrepância entre as exigências do cargo e os recursos individuais disponíveis para atendê-las (Ahola et al., 2014). O transtorno se manifesta pela perda progressiva das expectativas, da satisfação 
e do comprometimento no trabalho, associando-se à presença de um autoconceito negativo e atitudes desfavoráveis em relação ao trabalho e às pessoas do ambiente laboral, que inclui tanto os colegas de trabalho quanto os assistidos (Borgogni, Consiglio, Alessandri, \& Schaufeli, 2012; Tamayo \& Tróccoli, 2009).

O burnout é descrito como a manifestação concomitante de três fatores principais: (a) exaustão emocional, variável que diz respeito à vivência de sentimentos de desesperança, solidão, depressão, raiva, impaciência, irritabilidade, tensão, diminuição de empatia, sensação de baixa energia, fraqueza, preocupação; (b) despersonalização, aspecto que corresponde a distanciamento afetivo, ceticismo ou cinismo em relação às pessoas com as quais se relaciona, vivenciados por meio de sensação de alienação em relação aos outros, sendo a sua presença muitas vezes desagradável e não desejada; e (c) realização profissional, variável que corresponde ao nível de percepção da eficácia profissional, ligada a valores profissionais de trabalho e satisfação com atividade ocupacional (Trigo et al., 2007). Na direção de aprimoramento do modelo teórico de burnout, Borgogni et al. (2012) introduzem uma nova dimensão sobre o transtorno, a de tensão interpessoal (interpesonal strain), relacionada ao desconforto e ao desengajamento com as pessoas do trabalho. Em um estudo com staff hospitalar na Itália, Consiglio (2014) verificou tal processo associado a diferentes níveis de relacionamento no trabalho, como, chefia, colegas e clientela.

O instrumento utilizado com mais frequência para identificação do burnout é o Inventário de Burnout de Maslach (Maslach \& Jackson, 1981; Thalhammer \& Paulitsch, 2014), amplamente validado no mundo, inclusive no Brasil (Carlotto \& Câmara, 2004; Lautert, 1995). Entretanto, em pesquisas brasileiras, utiliza-se também para avaliação do referido construto o instrumento denominado de Escala de Caracterização do Burnout (Tamayo \& Tróccoli, 2009). Ambas as ferramentas apresentam indicadores favoráveis de validade e fidedignidade.

Estudos realizados com profissionais de saúde em vários países sugerem correlações entre a atividade de enfermeiros, médicos e outros profissionais de hospitais e o burnout (Chou, Li, \& Hu, 2014; Jasperse, Herst, \& Dungey, 2014; Wisetborisut, Angkurawaranon, Jiraporncharoen, Uaphanthasath, \& Wiwatanadate, 2014), embora nem todos estejam igualmente submetidos ao transtorno, a depender, entre outros, da distribuição desigual dos recursos disponíveis para a ação, mesmo em atividades com alta demanda profissional (Heeb \& Haberey-Knuessi, 2014).

No que tange aos aspectos preditivos do burnout, destacam-se fatores organizacionais (p. ex., jornada de turnos, metas), sociais (p. ex., relacionamento com chefia, suporte social) e individuais (p. ex., autoeficácia, estresse, experiência profissional) (Chou et al., 2014; Wisetborisut et al., 2014), além da personalidade resistente (bardiness) e o clima organizacional (Gershon et al., 2007).

\section{Clima de segurança, condições e contexto de trabalho e burnout}

Clima de trabalho como construto geral corresponde as dimensões psicológicas percebidas da organização de trabalho, incluindo avaliações cognitivas e afetivas dos indivíduos sobre diferentes elementos objetivos e subjetivos da organização, como a percepção sobre relação com a chefia, organização do trabalho, normas e procedimentos, satisfação com o trabalho, relacionamento interpessoal com colegas, justiça organizacional, entre outros (Puente-Palacios \& Martins, 2013). Clima de segurança, por sua vez, é um conceito mais particular de clima organizacional, abordando os elementos cognitivos e afetivos, além do conjunto de percepções e práticas compartilhadas sobre o risco e a segurança no trabalho (Gershon et al., 2007; Zohar, 1980), sendo este um construto de bastante relevância para atividades ocupacionais onde ocorram exposições a diferentes agentes de risco, como instituições hospitalares, construção civil, entre outros.

De acordo com Cohen, Smith e Cohen (1975) e Díaz e Cabrera (1997), o clima de segurança positivo associa-se a ações de investimento em segurança no trabalho, predizendo resultados mais positivos em termos desse conceito. Clima negativo, por sua vez, relaciona-se com condutas inapropriadas de segurança, podendo contribuir para a exposição profissional a situações de intensificação do risco e a acidentes no contexto de trabalho. Em organizações hospitalares, o questionário de clima de segurança desenvolvido por Gershon et al. (2000) vem sendo utilizado em diferentes investigações sobre saúde e segurança no trabalho. O instrumento validado para o contexto brasileiro com base nessa ferramenta avalia as dimensões clima para programa e normas de segurança, clima de suporte para práticas de trabalho e segurança, clima sobre equipamento de segurança e organização do ambiente e clima para treinamento e educação em segurança (De Andrade, 2014). 
Já o conceito de condições de trabalho é utilizado por pesquisadores de diversas áreas que estudam o trabalho humano. Conforme Ferreira e Mendes (2008), o conceito de Contexto de Produção de Bens e Serviços (CPBS) apreende de maneira sistemática as dimensões material, organizacional e social da atividade de modo a não desarticular as variáveis que compõem as condições de trabalho (Ferreira \& Mendes, 2008). Para mensurar as diferentes características do CPBS, desenvolveu-se uma escala psicométrica denominada Escala de Avaliação do Contexto de Trabalho, que avalia as seguintes dimensões: (a) a organização do trabalho, que corresponde às concepções e práticas de gestão de pessoas e do trabalho; (b) as condições de trabalho, correspondentes aos elementos estruturais que caracterizam a infraestrutura e o apoio institucional em situação de produção; e (c) relações socioprofissionais, caracterizando a dimensão social do contexto de trabalho. Nesta pesquisa lança-se mão dessa perspectiva por permitir melhor sistematização das diversas dimensões que compõem o contexto do trabalho.

Foi constatado que do burnout e seus preditores resultam diferentes consequências à saúde dos trabalhadores, bem como para os comportamentos de segurança dos profissionais e seus resultados operacionais e clínicos (Profit et al., 2014). Entretanto, no que tange aos aspectos da segurança profissional dos trabalhadores de saúde, poucos estudos investigam os elementos de cultura de segurança (Sexton et al., 2014), e menos ainda, o clima de segurança e suas relações com o burnout (Profit et al., 2014), mesmo que haja indícios de relações entre o burnout, a qualidade do serviço prestado, a sua segurança e a dos pacientes (Halbesleben, Wakefield, Wakefield, \& Cooper, 2008; Montgomery, Panagopoulou, Kehoe, \& Valkanos, 2011; Montgomery, Todorova, Baban, \& Panagopoulou, 2013). Foi proposto um modelo visando articular aspectos das condições de trabalho, em particular a cultura organizacional, a qualidade do serviço e o burnout (Montgomery et al., 2011). No entanto, ainda não há um modelo específico articulando questões de segurança no trabalho e variáveis do contexto de trabalho, do clima de segurança e o burnout. Visando a contribuir para essa carência, realizou-se esta pesquisa, que teve por objetivo avaliar diferentes aspectos psicossociais do trabalho em contexto hospitalar e seus efeitos na manifestação da síndrome do burnout. Da mesma forma, procurou-se desenvolver um modelo teórico estrutural explicativo relacionando variáveis dimensionais de trabalho, burnout e saúde.

\section{MÉTODO}

\section{Participantes}

A amostra deste estudo possui caráter de conveniência e contou com 200 profissionais da área da saúde, dos quais 174 eram do sexo feminino (87\%), e a média de idade foi de 34,4 anos ( $D P=8,71$ anos). Em termos de aspectos profissionais, a média de anos trabalhados foi de 6,5 anos $(D P=6,83$ anos). Em relação às categorias profissionais que constituíram a amostra, houve maior prevalência de técnicos em enfermagem $(n=139$; $69,5 \%)$, seguida por enfermeiros $(n=14 ; 7,0 \%)$, médicos $(n=12 ; 6,0 \%)$ e categoria geral, incluindo psicólogos, fisioterapeutas e terapeutas ocupacionais $(n=35 ; 17,5 \%)$.

\section{Instrumentos}

Para a coleta de dados, utilizou-se um conjunto de questionários, apresentados na ordem em que são descritos abaixo.

1. Questionário sociodemográfico: instrumento com itens de caracterização dos participantes (sexo, idade, escolaridade, hospital, atividade fim, histórico de exposição a acidentes, etc.).

Quanto aos aspectos de saúde e variáveis psicossociais, incluíram-se as seguintes medidas:

2. Inventário de Burnout de Malasch (MBI) - escala de 22 itens que avalia como o trabalhador percebe a vivência do seu trabalho a partir de três dimensões conceituais: (a) exaustão emocional, (b) realização profissional e (c) despersonalização. $\mathrm{Na}$ interpretação dos subfatores de burnout, escores mais elevados em cada dimensão correspondem a maior intensidade das emoções e respostas do trabalhador. $\mathrm{O}$ inventário foi traduzido e adaptado apresentando indicadores de validade e confiabilidade favoráveis (Carlotto \& Câmara, 2004).

3. Escala de Avaliação do Contexto de Trabalho (EACT) - o instrumento com 30 itens que acessa três dimensões: (a) organização do trabalho, avalia as concepções e práticas de gestão de pessoas e do trabalho $(\alpha=0,72)$; (b) condição de trabalho, corresponde aos elementos estruturais que caracterizam a infraestrutura e o apoio institucional para execução da atividade de trabalho $(\alpha=0,89)$; e (c) relações 
socioprofissionais, caracteriza os sociais e interpessoais do contexto de trabalho $(\alpha=0,87)$ (Ferreira $\&$ Mendes, 2008).

4. Escala de clima de segurança (CLIMA-SEG) - medida validada para contexto brasileiro por De Andrade (2014), tomando por base o modelo de Gershon et al. (2000). O instrumento possui 34 itens dispostos em uma estrutura de quatro fatores: (a) programa e normas de segurança, (b) suporte para práticas de trabalho e segurança, (c) equipamento de segurança e organização do ambiente e (d) treinamento e educação em segurança. Todas as dimensões com índices de confiabilidade alfa de Cronbach superiores a 0,80 .

No que diz respeito às medidas de clima de segurança e condições de trabalho, os valores computados na apuração de resultados referenciam uma interpretação crescente para percepção positiva do clima de segurança, ou seja, escores baixos (na direção do valor 1) representam aspectos negativos do ambiente psicológicos e físico do trabalho; e escores elevados (na direção do valor 5), aspectos positivos e favoráveis do ambiente psicológicos e físico do trabalho.

5. Questionário geral de saúde (QSG-12) - Constitui-se na versão reduzida do General Health Questionnaire (Goldberg, 1972), com 12 itens que têm como objetivo a mensuração das dimensões ansiedade e depressão (Gouveia et al., 2003). Quanto maior o escore do indivíduo no fator, maior o nível de experimentação dos sintomas correspondentes. Os índices de confiabilidade observados na amostra brasileira foram 0,81 (Depressão) e 0,66 (Ansiedade).

\section{Procedimentos de coleta de dados e cuidados éticos}

Em conformidade com as normas do Conselho Nacional de Saúde, primeiramente foi analisada e aprovada a viabilidade do estudo via avaliação do Comitê de Ética em Pesquisa com Seres Humanos (CEPSH) da Universidade Federal do Espírito Santo (parecer número 395.290). Na sequência foram realizados contatos com hospitais públicos e privados de uma capital localizada na região sudeste, para solicitar a colaboração e permissão para coleta de dados com seus respectivos funcionários. Fornecida a permissão, as datas de coleta foram agendadas com a equipe do hospital. Destaca-se nesse momento a dificuldade de entrada e a burocracia de exigências em algumas instituições, sendo possível contar na amostra com apenas quatro hospitais.

O procedimento de coleta de dados foi realizado dentro dos estabelecimentos hospitalares, com aplicações coletivas dos questionários em ambientes destinados ao treinamento dos funcionários. A aplicação dos instrumentos foi feita por assistentes de pesquisa devidamente treinados no procedimento de coleta de dados e com instruções sobre procedimentos de segurança hospitalar (fornecidos pelo primeiro autor do artigo em parceria com o setor de pessoal de um dos hospitais da amostra). Os questionários foram aplicados em pequenos grupos organizados pelos setores de departamento pessoal e/ou recursos humanos.

No momento da aplicação os participantes foram informados do caráter voluntário e sigiloso de sua participação na pesquisa. Aqueles que se voluntariaram foram instruídos a ler e assinar o termo de consentimento livre e esclarecido, para que fosse possível utilizar suas respostas na pesquisa. Uma cópia do termo foi fornecida a cada participante, contendo informações sobre a pesquisa e o contato do pesquisador responsável e do comitê de ética, para eventuais dúvidas futuras sobre a pesquisa. Outra cópia do termo foi devidamente assinada e entregue aos assistentes de pesquisa no encerramento do questionário. O tempo de aplicação variou entre 15 e 25 minutos e, após isso, os devidos agradecimentos foram direcionados aos participantes.

\section{Procedimentos de análise de dados}

Os dados da pesquisa foram analisados com auxílio dos programas SPSS (versão 18) e Amos (versão 7.0). O trabalho não teve por objetivo uma análise diagnóstica do burnout no contexto das instituições investigadas, mas uma análise dos aspectos divergentes e convergentes dos construtos avaliados. Para tal, realizaram-se procedimentos de correlação (coeficiente de Pearson) e, para compreender o papel preditivo e as determinantes relacionais das subdimensões de burnout, executaram-se análises de regressão linear múltipla, tomando as variáveis de burnout como variáveis dependentes (exaustão emocional, despersonalização e realização) e as variáveis de clima e condições de trabalho como independentes.

Dentro do estudo também foram estruturados dois modelos de equações estruturais, visando a um entendimento causal das variáveis. Com base em Byrne (2010) e Hair, Anderson, Tatham e Black (2005), foram tomados os indicadores de ajuste dos modelos descritos a seguir: 
- $\quad \chi^{2}$ (qui-quadrado): indicador que avalia a probabilidade do modelo em se ajustar aos dados da matriz.

- $\quad \chi^{2} / g l$ indicador de ajustamento em que são recomendados valores entre 2 e 3 , admitindo-se até 5 .

- CFI (Comparative Fit Index): indicador comparativo sobre o ajuste dos modelos cujos valores variam de 0 a 1 , e quanto mais próximos de 1 , melhor é o ajuste, sendo valores superiores a 0,90 adotados como indicadores da qualidade do modelo.

- RMSEA (Root Mean Square Error): indicador de resíduos em que se estima valores situados entre 0,05 e 0,08, com intervalo de confiança de 90\%, aceitando-se valores de até 0,10.

- GFI (Goodness-of-fit Index) e AGFI (Adjusted Goodness-of-fit Index): indicadores do ajuste ponderado relacionados com proporção de variância-covariância nos dados explicada pelo modelo, com valores variando de 0 a 1 e valor de parâmetro próximo ao intervalo de 0,90 a 0,95 .

- $\lambda$ (Coeficiente lambda): indicador do procedimento de regressão, com resultados que variam de 0 a 1 (valores mais próximos de 1 sinalizam melhor desempenho de predição da variável para o modelo).

\section{RESULTADOS}

\section{Burnout e variáveis de trabalho}

Com o objetivo de verificar aspectos associados à relação das dimensões de burnout com aspectos psicossociais do trabalho (clima de segurança e suas dimensões) e variáveis ligadas à percepção de condições de trabalho (condições organizacionais, físicas e socioprofissionais do trabalho), executou-se uma análise de correlações entre as diferentes variáveis.

A Tabela 1 apresenta os resultados dessa análise.

TABELA 1. Matriz de correlações de dimensões de burnout, escala de condições de trabalho e clima de segurança

\begin{tabular}{|c|c|c|c|c|c|c|c|c|c|c|}
\hline & EE & $\mathbf{R P}$ & DE & СТ Org & $\begin{array}{l}\text { CT Físi- } \\
\text { ca }\end{array}$ & $\begin{array}{l}\text { CT Soc } \\
\text { Inter }\end{array}$ & $\begin{array}{l}\text { Clima } \\
\text { PNS }\end{array}$ & $\begin{array}{l}\text { Clima } \\
\text { SPTS }\end{array}$ & $\begin{array}{l}\text { Clima } \\
\text { ESOA }\end{array}$ & $\begin{array}{l}\text { Clima } \\
\text { TDS }\end{array}$ \\
\hline E.E. & 1 & & & & & & & & & \\
\hline R.P. & $-0,04$ & 1 & & & & & & & & \\
\hline D.E & $0,40^{\star \star}$ & $-0,25^{\star *}$ & 1 & & & & & & & \\
\hline СT Org & $-0,51^{\star *}$ & $0,15^{*}$ & $-0,37^{\star *}$ & 1 & & & & & & \\
\hline CT Física & $-0,41^{\star *}$ & 0,11 & $-0,22^{\star *}$ & $0,67^{\star \star}$ & 1 & & & & & \\
\hline CT Soc.Inter & $-0,46^{\star \star}$ & $0,25^{\star *}$ & $-0,40^{\star *}$ & $0,67^{* *}$ & $0,64^{\star *}$ & 1 & & & & \\
\hline Clima PNS & $-0,28^{\star \star}$ & 0,03 & $-0,19^{\star \star}$ & $0,32^{\star \star}$ & $0,45^{\star \star}$ & $0,46^{\star \star}$ & 1 & & & \\
\hline Clima SPTS & $-0,49^{\star *}$ & $0,19^{* *}$ & $-0,36^{\star *}$ & $0,56^{\star *}$ & $0,50^{\star *}$ & $0,66^{\star \star}$ & $0,48^{\star *}$ & 1 & & \\
\hline Clima ESOA & $-0,31^{\star *}$ & $0,12^{*}$ & $-0,21^{\star *}$ & $0,33^{* *}$ & $0,44^{\star \star}$ & $0,35^{\star \star}$ & $0,54^{* *}$ & $0,46^{\star \star}$ & 1 & \\
\hline Clima TDS & $-0,20^{\star *}$ & 0,11 & $-0,13^{*}$ & $0,26^{\star *}$ & $0,39^{\star *}$ & $0,36^{\star \star}$ & $0,67^{* *}$ & $0,50^{\star *}$ & $0,60^{\star *}$ & 1 \\
\hline
\end{tabular}

Nota. EE. (exaustão emocional); RP (realização profissional); DE (despersonalização); CT. Org. (condição de trabalho organizacional); CT. Física (condição de trabalho física); CT Soc. Inter. (condição de trabalho socioprofissional); Clima PNS (programa e normas de segurança); Clima SPTS (suporte para práticas de trabalho e segurança); Clima ESOA (equipamento de segurança e organização do ambiente); Clima TDS (treinamento e educação em segurança).

A partir da Tabela 1 observam-se correlações significativas e elevadas entre diferentes dimensões de burnout (exaustão emocional, realização profissional e despersonalização) com dimensões clima de segurança (programa e normas de segurança, suporte para práticas de trabalho e segurança, equipamento de segurança e organização do ambiente e clima de treinamento e educação em segurança) e aspectos de condições de trabalho (condição da organização do trabalho, condição de física do trabalho e condição socioprofissional).

De forma específica, a dimensão de burnout exaustão emocional correlacionou-se negativamente a: condição da organização do trabalho $(r=-0,51)$; condição de física do trabalho $(r=-0,41)$; condição socioprofissional $(r=-0,46)$; programa e normas de segurança $(r=-0,28)$, suporte para práticas de trabalho e segurança $(r=-0,49)$, equipamento de segurança e organização do ambiente $(r=0,31)$; treinamento e educação em segurança $(r=-0,20)$; e positivamente com a dimensão despersonalização $(r=0,40)$.

$\mathrm{O}$ aspecto de realização profissional do construto burnout, por sua vez, correlacionou-se positivamente a: condição socioprofissional $(r=0,25)$; suporte para práticas de trabalho e segurança $(r=0,20)$, equipamento 
de segurança e organização do ambiente $(r=0,13)$ e; satisfação com a vida $(r=0,20)$; e negativamente com a dimensão despersonalização $(r=-0,25)$. Por último, a variável despersonalização correlacionou-se negativamente a: condição da organização do trabalho $(r=-0,37)$; condição de física do trabalho $(r=-0,22)$; condição socioprofissional $(r=-0,40)$; programa e normas de segurança $(r=-0,19)$, suporte para práticas de trabalho e segurança $(r=-0,36)$, equipamento de segurança e organização do ambiente $(r=-0,21)$; treinamento e educação em segurança $(r=-0,13)$; satisfação com a vida $(r=-0,25)$.

\section{Preditores de burnout}

Para analisar as dimensões preditoras de burnout, consideraram-se nessa seção os construtos de clima de segurança e condições de trabalho como variáveis independentes, e os construtos dimensionais de burnout (exaustão emocional, realização profissional e despersonalização), como variáveis dependentes. No total, foram realizadas três análises de regressão linear múltipla (Tabela 2).

TABELA 2. Preditores de burnout

\begin{tabular}{|c|c|c|c|c|c|c|c|c|c|c|c|c|}
\hline & \multicolumn{4}{|c|}{ Exaustação emocional $\left(R^{2}=34,1 \%\right)$} & \multicolumn{4}{|c|}{ Realização profissional $\left(R^{2}=9,0 \%\right)$} & \multicolumn{4}{|c|}{ Despersonalização $\left(R^{2}=21,0 \%\right)$} \\
\hline & $B$ & $S E$ & $\beta$ & $p$ & $B$ & $S E$ & $\beta$ & $p$ & $B$ & $S E$ & $\beta$ & $p$ \\
\hline CT. Org. & $-0,43$ & 0,14 & $-0,28$ & $<0,01$ & $-0,02$ & 0,10 & $-0,02$ & 0,80 & $-0,22$ & 0,09 & $-0,21$ & 0,02 \\
\hline CT. Física & $-0,03$ & 0,10 & $-0,03$ & 0,74 & $-0,05$ & 0,07 & $-0,07$ & 0,46 & 0,13 & 0,06 & 0,19 & 0,05 \\
\hline CT. Soc. & $-0,08$ & 0,12 & $-0,06$ & 0,54 & 0,25 & 0,09 & 0,30 & 0,00 & $-0,22$ & 0,08 & $-0,26$ & 0,01 \\
\hline Clima PNS & $-0,08$ & 0,13 & $-0,06$ & 0,47 & $-0,15$ & 0,08 & $-0,21$ & 0,03 & $-0,02$ & 0,08 & $-0,02$ & 0,78 \\
\hline Clima SPTS & $-0,41$ & 0,13 & $-0,27$ & $<0,01$ & 0,05 & 0,09 & 0,05 & 0,56 & $-0,13$ & 0,09 & $-0,14$ & 0,13 \\
\hline Clima ESOA & $-0,19$ & 0,13 & $-0,11$ & 0,14 & 0,09 & 0,09 & 0,08 & 0,33 & $-0,11$ & 0,09 & $-0,11$ & 0,20 \\
\hline Clima TDS & 0,21 & 0,13 & 0,15 & 0,09 & 0,09 & 0,09 & 0,10 & 0,33 & 0,09 & 0,09 & 0,10 & 0,29 \\
\hline
\end{tabular}

Nota. CT. Org. (condição de trabalho organizacional); CT. Física (condição de trabalho física); CT Soc. Inter. (condição de trabalho socioprofissional); Clima PNS (programa e normas de segurança); Clima SPTS (suporte para práticas de trabalho e segurança); Clima ESOA (equipamento de segurança e organização do ambiente); Clima TDS (treinamento e educação em segurança); $R^{2}$ (representa a proporção da variância explicada pela VD).

No primeiro modelo testado para explicar exaustão emocional, as variáveis de clima e condições de trabalho predisseram $34,1 \%$ da variância do construto $[F(7,19)=14,17, p<0,01]$, sendo preditores significativos as variáveis condição de trabalho organizacional e clima suporte para práticas de trabalho e segurança. $\mathrm{O}$ segundo modelo, para predizer realização profissional, teve no conjunto de suas variáveis $9 \%$ de explicação $[F(7,19)=2,74, p<0,01]$, sendo significativas as variáveis condição de trabalho socioprofissional e clima de programa e normas de segurança. Por fim, o terceiro modelo, visando a explicar a variável despersonalização, obteve, no conjunto de variáveis de entrada, $21 \%$ de explicação do modelo $[F(7,19)=7,30, p<0,01]$, sendo preditores significativos as variáveis condição de trabalho organizacional, condição de trabalho física e condição de trabalho socioprofissional.

\section{Modelos psicossociais de burnout}

Com objetivo final de compreender a relação concomitante das variáveis deste estudo, estruturaram-se dois modelos a partir da técnica estatística de modelagem de equações estruturais - SEM (Byrne, 2010). Observase que, para estruturação dos modelos de SEM, optou-se, para a condução deste estudo, pela adoção das variáveis de clima de segurança em duas dimensões (programa e normas de segurança e suporte para práticas de trabalho e segurança). 


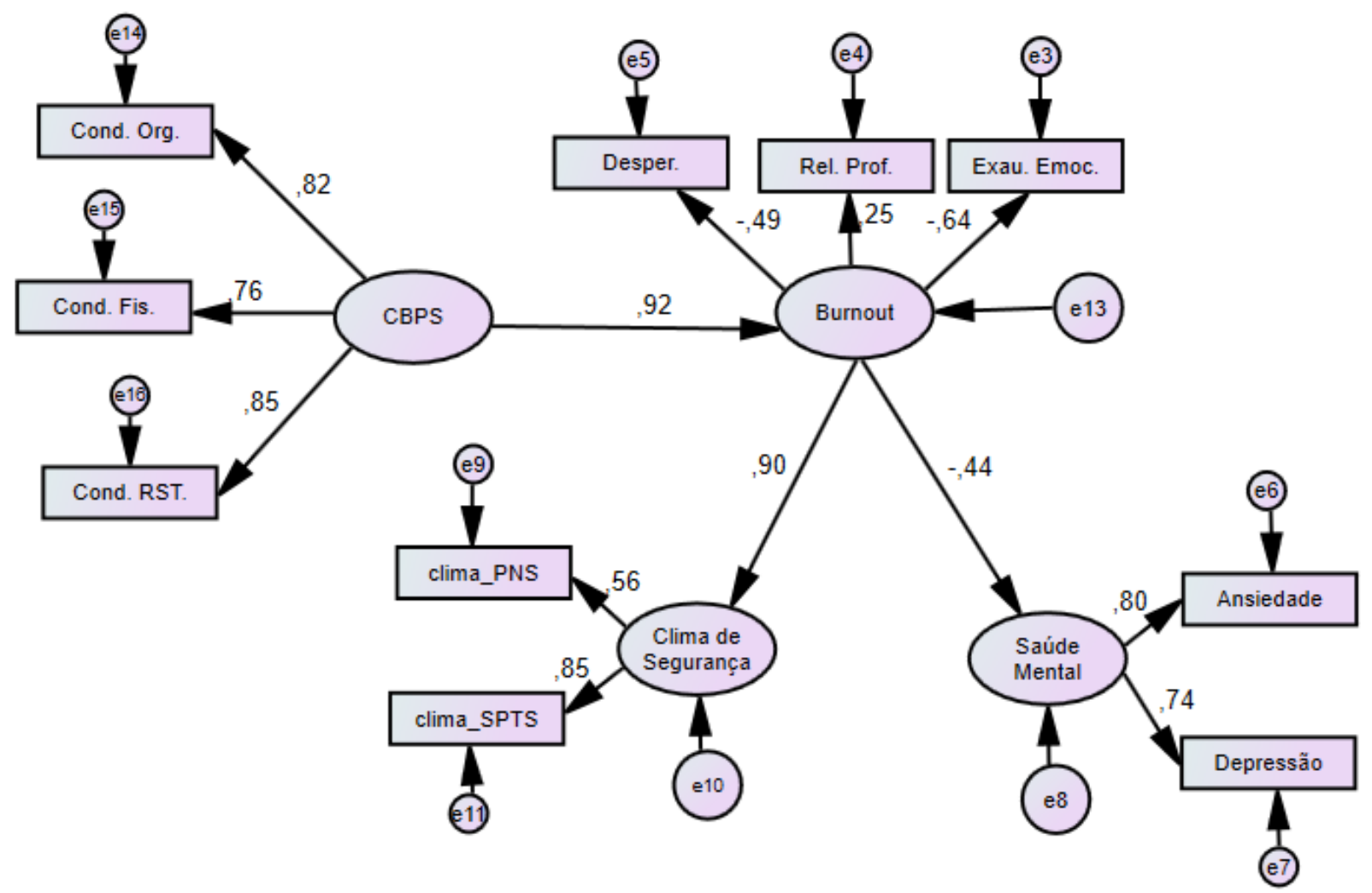

MODELO 2

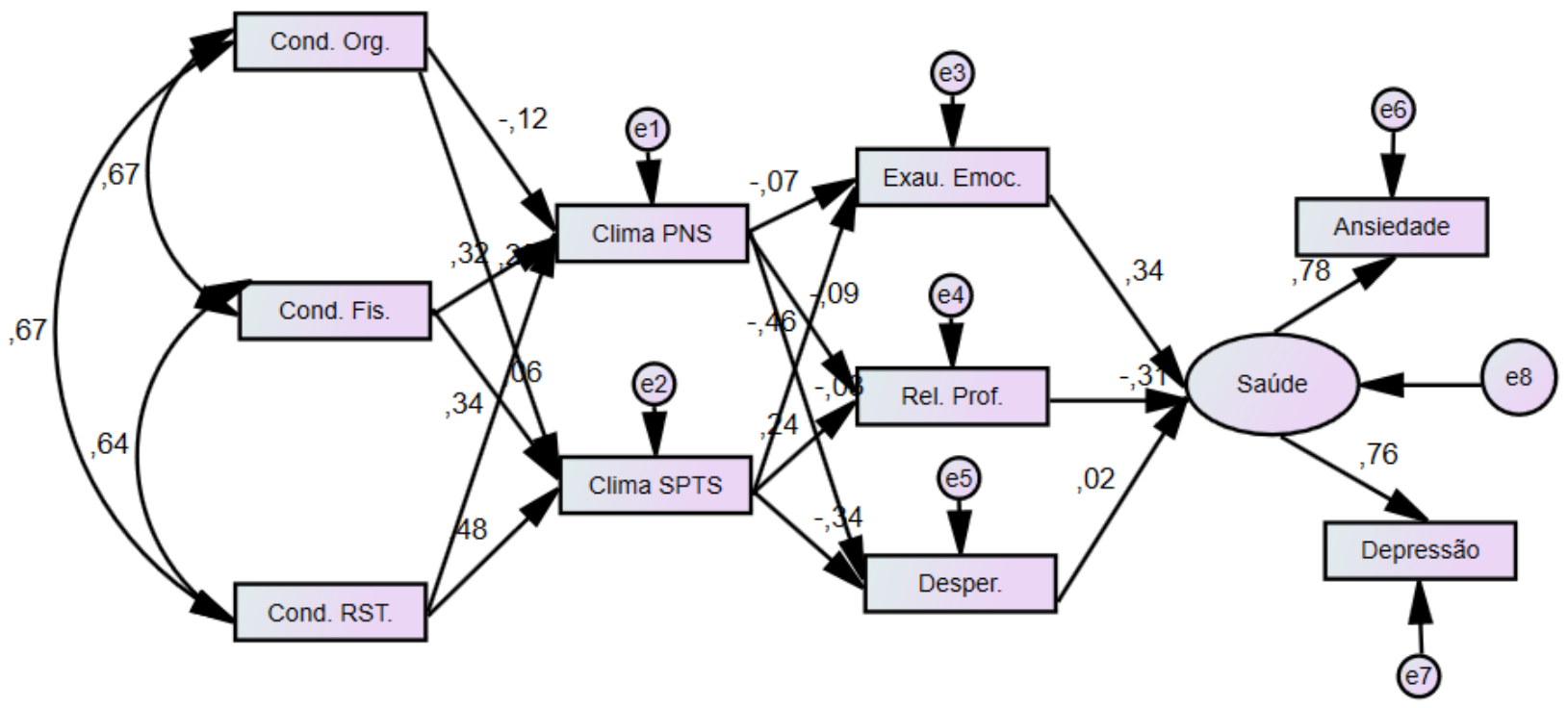

FIGURA 1. Modelos psicossociais de trabalho, burnout e saúde

O primeiro modelo testado, Modelo 1 apresenta causalidade em dois níveis: condições de trabalho (CPBS), afetando aspectos de burnout, os quais operam como variáveis causadoras do processo de saúde (nível experimentado de sintomas de ansiedade e depressão); e aspectos de clima de segurança no trabalho (representado pelas subdimensões do construto: programa e normas de segurança - Clima PNS, e suporte para práticas de trabalho e segurança - Clima SPTS).

O Modelo 2 apresenta causalidade em quatro níveis: condições de trabalho (física, organizacional e socioprofissional), afetando aspectos específicos da percepção de clima de segurança (programa e normas de segurança-Clima PNS; suporte para práticas de trabalho e segurança-Clima SPTS), que, por sua vez, afeta 
aspectos dimensionais da percepção de burnout (exaustão emocional, realização profissional e despersonalização), as quais operam como variáveis de efeito em termos do processo de saúde. $\mathrm{Na}$ questão de diferenças do primeiro e do segundo modelo, tem-se no primeiro o burnout se relacionando com aspectos de clima de segurança e saúde e, no Modelo 2, a variável clima de segurança, operando como construto intermediário entre fatores de condições de trabalho e aspectos de burnout e, posteriormente, saúde.

Os índices de ajuste dos modelos foram favoráveis em praticamente todos os indicadores. No primeiro modelo, os índices foram $\left[\chi^{2}=78,08, g l=32(p<0,01), \chi^{2} / g l=2,44, \mathrm{RMR}=0,03\right.$, GFI=0,93, AGFI=0,88, CFI=0,93, RMSEA $(90 \% \mathrm{CI})=0,08(0,06-0,10)]$. Com relação ao segundo modelo, os indicadores também foram adequados: $\left[\chi^{2}=90,39, g l=25(p<0,01), \chi^{2} / g l=3,61, \mathrm{RMR}=0,05\right.$, GFI=0,91, AGFI=0,81, CFI=0,98, RMSEA $(90 \% \mathrm{CI})=0,11(0,09-0,14)]$. Para verificar qual modelo possuiu melhor ajuste, optou-se pelo procedimento do delta qui-quadrado: $\left[\Delta \chi^{2}(2)=12,30, p<0,01\right]$, sugerindo o modelo 1 como o mais adequado.

\section{DISCUSSÃO}

A principal contribuição deste estudo é a proposição de modelos teóricos integrativos de variáveis psicossociais, clima de segurança e saúde para trabalhadores em contexto hospitalar. Procurando compreender a relação concomitante das variáveis do estudo, testaram-se modelos de equações estruturais. Conforme (Byrne, 2010), a modelagem é uma modalidade multivariada de análise de dados que permite a avaliação simultânea de múltiplos construtos. Os dois modelos propostos neste estudo são considerados inovadores, propondo a junção de quatro construtos e suas subdimensões na explicação de um quadro contextual de variáveis.

Destacando o primeiro modelo testado, pode-se constatar que o fenômeno burnout, como compreendido neste estudo, não é apenas uma consequência do trabalho nos hospitais, mas também um mediador das relações entre o contexto de trabalho e o clima de segurança. A partir dos presentes resultados, percebe-se que o burnout, compreendido como efeito do contexto do trabalho, impacta tanto aspectos do clima de segurança como variáveis individuais de saúde (ansiedade e depressão). Tais indicadores corroboram estudos que demonstram correlações entre burnout e clima de segurança (Profit et al., 2014) e cultura organizacional, mas também sugerem um modelo de causalidade dessa relação.

Resultam desse modelo consequências importantes, sobretudo na direção de que o controle do burnout não visa apenas à qualidade de vida dos trabalhadores, mas também à melhoria do clima de segurança, à consequente redução de acidentes de trabalho e à possibilidade de melhoria da qualidade da prestação de serviços hospitalares, conforme demonstrado em outros modelos (Montgomery et al., 2011). Se a extensão do efeito dessa relação na quantidade de episódios de acidente não foi verificada nesta pesquisa, outros estudos demonstram a relação entre clima de segurança e acidentes (Díaz \& Cabrera, 1997).

Sendo assim, o modelo aqui proposto pode auxiliar a compreensão ampliada da relação entre contexto de trabalho e acidentes, sendo estes últimos, em hipótese e direção futura de pesquisa, mediados pelas condições de saúde relacionadas ao trabalho dos profissionais (burnout) e pelos efeitos e percepções psicossociais (clima) que o contexto e as condições de saúde produzem neles.

A avaliação e o tratamento do burnout, por meio de intervenções no contexto de trabalho, podem contribuir para a melhoria da saúde dos trabalhadores e a redução de acidentes de trabalho. Com base nas correlações apresentadas neste estudo, verifica-se que a exaustão emocional é fortemente associada aos três aspectos de condição de trabalho (organizacional, físico e social/interpessoal). Se dá destaque para a variável organizacional, a qual se mostrou preditora também nas análises de regressão, demonstrando a importância dos diferentes aspectos do planejamento e das prescrições do trabalho no transtorno de burnout.

$\mathrm{Na}$ mesma direção associam-se aspectos de clima de segurança, tendo maior relevância a variável suporte para práticas de trabalho e segurança. Em relação à dimensão de realização profissional, observam-se correlações mais fracas com aspectos de condições de trabalho, destacando entre essas a variável socioprofissional quanto às variáveis de clima de segurança. $\mathrm{Na}$ ordem dos aspectos interpessoais ou condições sociprofissionais, os estudos recentes de Consiglio (2014) salientam que as tensões interpessoais ligadas ao trabalho são proeminentes de burnout, além de produzirem outros efeitos negativos na saúde dos indivíduos e deteriorações em contextos externos à vida socioprofissional, como nas relações familiares (Suñer-Soler et al., 2014).

O aspecto de despersonalização, por sua vez, correlacionou-se fortemente com condições de trabalho nas dimensões organizacional e socioprofissional, sendo a dimensão de clima para suporte para práticas de trabalho e segurança a relação de mais força dessa dimensão. Esses resultados apontam que a saúde dos trabalhadores 
(burnout, depressão e ansiedade) está relacionada tanto a fatores psicossociais (clima de segurança e de suporte social) quanto a fatores físicos e organizacionais do trabalho (Frasquilho, 2005; Moreno, Gil, Haddad, \& Vannuchi, 2011).

A correlação elevada entre o clima de suporte e o contexto interacional de trabalho $(r=0,50)$ sinaliza que as percepções positivas de relações pessoais e de trabalho afetam diretamente as percepções positivas do clima de suporte para práticas e segurança. A relação entre burnout e fatores psicossociais e organizacionais corroboram resultados de outros estudos (Chou et al., 2014), demonstrando a complexidade de fatores em torno dos processos de adoecimento dos trabalhadores. Dentro deste estudo, clima de suporte foi a dimensão mais correlacionada a todos os fatores do contexto de trabalho, reforçando o fato de que a percepção de suporte possui papel fundamental para a exaustão emocional do burnout, evidenciando relações semelhantes às encontradas nos estudos de estresse, que demonstram o papel crucial do suporte social como fator protetor ao transtorno (Alves, Chor, Faerstein, Lopes, \& Werneck, 2004; Tamayo \& Tróccoli, 2002).

Pode-se compreender, nesta exposição, que a abordagem da saúde dos profissionais dessa área necessita englobar, entre outras, as dimensões psicossociais do processo saúde-doença, onde as construções psicossociais, organizacionais e culturais sejam apreendidas, sem apagar simultaneamente as necessidades individuais, uma vez que a confluência permite maior motivação profissional, equilíbrio mental, bom humor e satisfação no desempenho das atividades cotidianas (Madureira, 2012; Minayo-Gomez \& Thedim-Costa, 1997).

Se os modelos aqui propostos sugerem que o burnout impacta tanto na saúde como no clima de segurança, observa-se que, entre os aspectos preditivos de suas facetas, o contexto de trabalho exerce também papel fundamental nesse processo. O modelo mais explicativo a partir das variáveis do estudo foi o da exaustão emocional, sendo significativas as variáveis condição de trabalho organizacional, clima de segurança de suporte para práticas de trabalho e segurança e clima de treinamento e educação em segurança. Esses dados corroboram os de outras pesquisas, sugerindo que aspectos organizacionais são preditores do burnout (Jasperse et al., 2014). Essa relação sinaliza que a baixa percepção de clima de suporte para práticas e trabalho em segurança intensifica a percepção de esforços necessários que cada trabalhador terá que realizar individualmente para conseguir executar suas tarefas.

Não por acaso, a exaustão emocional também é a condição mais diretamente relacionada ao contexto do trabalho. Aliás, no que tange aos preditores de burnout, são exatamente esses fatores os mais significativos do transtorno, embora também sejam importantes os do contexto de trabalho. O segundo modelo de maior porcentagem de explicação foi o de despersonalização, sendo significativamente preditivas apenas as variáveis relacionadas às condições de trabalho (organizacional, físico e socioprofissional). O modelo de realização profissional foi o menos explicativo, sendo significativas apenas as variáveis condição de trabalho socioprofissional e climas de programa e normas de segurança.

A natureza inovadora do modelo se depara com limitações metodológicas que envolvem, entre outros aspectos, a natureza da amostra, composta apenas por colaboradores de uma grande região de um estado do sudeste e sua não representação probabilística por hospitais e número de colaboradores. Outro aspecto sobre limitação amostral diz respeito ao fato de ela ser composta por profissionais de diferentes categorias que trabalham em hospital, o que, pela sua variação, pode interferir na precisão dos dados obtidos. Essa precisão também pode ter sido afetada pela amostra ser constituída por profissionais que trabalham em hospitais públicos e privados que têm diferentes modos de funcionamento, tipos de contrato trabalhista e organização de trabalho.

Com respeito ao aspecto de amostragem em instituições hospitalares, sejam elas públicas ou privadas, observa-se muitas vezes receio por parte dos colaboradores dessas organizações para expressar suas opiniões em pesquisa que envolvam algum tipo de avaliação sobre aspectos do trabalho, além, é claro, da pouca participação ou envolvimento de gestores e responsáveis pelos recursos humanos. Torna-se importante maior compromisso e interesses dos diferentes membros dessas organizações para estudos futuros com maior representatividade.

A despeito dessas observações, a amostra desta pesquisa teve problemas nesses aspectos, mas os resultados obtidos devem ser considerados já que o trabalho elaborado em um contexto permeado de desafios, estresse e sofrimento necessita ser envolvido por um estímulo que permita sua continuidade. Na avaliação geral dos proponentes desta pesquisa, as barreiras e burocracias impostas por instituições hospitalares não desmerecem a natureza propositiva de um modelo que oferece elementos para exploração e confirmação em futuras pesquisas, considerando-se o novo modelo integrador que tomou como unidade de base modelos teóricos já confirmados. 
Uma sugestão para futuros pesquisadores é a de que a exploração de modelos que integrem outras questões destacadas por diversos autores, como a falta de reconhecimento profissional (Shimizu \& Ciampone, 1999; Sprandel \& Vaghetti, 2012), a intenção de desistir da profissão (Suñer-Soler et al., 2014) e o conflito trabalhofamília (Slanetz, 2011), sejam interessantes para o avanço do conhecimento tanto sobre o burnout como sobre as diferentes pressões psicossociais as quais são submetidos os trabalhadores da área da saúde.

Pesquisas com enfoque em problemas reais do mundo do trabalho contribuem para a perspectiva de que as políticas de prevenção de acidentes devam ser ampliadas para estratégias mais complexas que as tradicionais práticas de educação e informação. Ressalta-se a importância de intervenções amplas em relação ao contexto do trabalho, corroborando estudos que apontam que intervenções limitadas, como apenas alteração na jornada de trabalho, as quais não repercutem reduções tão expressivas no risco de burnout (Richter, Kostova, Baur, \& Wegner, 2014).

Conclui-se o presente trabalho reforçando a importância de pesquisas que envolvam fenômenos ligados à promoção e à proteção da saúde dos profissionais do contexto hospitalar. Destaca-se o papel institucional e de gestores do poder público para que esses elementos possam ocorrer de forma eficaz, envolvendo diretamente a motivação de gestores para avaliação e implementação de mudanças que possam atender às necessidades e à natureza dos serviços de prestação de cuidado de cada paciente na rotina diária do ambiente hospitalar.

A realização deste estudo, exemplo de parceria entre instituições hospitalares e pesquisadores, sinaliza como resultados associações entre aspectos de saúde (burnout), organização do trabalho (CPBS) e percepções por parte dos trabalhadores dos agentes de risco do ambiente de trabalho (clima de segurança). $\mathrm{O}$ estudo resultou em informações importantes para orientação de mudanças e conhecimento para futuros estudos no âmbito dos fenômenos em questão.

Compartilha-se o compromisso e motiva-se futuros pesquisadores no desafio de conhecer as diferentes nuances do trabalho, visando dentro da realidade de cada contexto sócio-histórico, compromisso de consciência e mudança que, se possível, conciliem os interesses tanto dos trabalhadores como das organizações de trabalho. Porém, cabe apontar que, na impossibilidade de atender a ambos, caberá a pesquisadores, gestores e trabalhadores definir sua prioridade de atuação, posicionando-se frente a eventuais contradições com as quais se depare.

\section{REFERÊNCIAS}

Ahola, K., Salminen, S., Toppinen-Tanner, S., Koskinen, A., \& Vaananen, A. (2014). Occupational burnout and severe injuries: An eight-year prospective cohort study among Finnish forest industry workers. Journal of Occupational Health, 55(6), 450-457. doi:10.1539/joh.13-0021-OA

Alves, M. G. M., Chor, D., Faerstein, E., Lopes, C. S., \& Werneck, G. L. (2004). Versão resumida da "job stress scale": Adaptação para o português. Revista de Saúde Pública, 38(2), 164-171. doi: 10.1590/S0034-89102004000200003

Amigo, I., Asensio, E., Menendez, I., Redondo, S., \& Ledesma, J. A. (2014). Working in direct contact with the public as a predictor of burnout in the banking sector. Psicothema, 26(2), 222-226. doi:10.7334/psicothema2013.282

Andrade, P. S., \& Cardoso, T. A. O. (2012). Prazer e dor na docência: Revisão bibliográfica sobre a síndrome de burnout. Saúde e Sociedade, 21(1), 129-140. doi:10.1590/S0104-12902012000100013

Bataile, S. (2014). La reconstruction professionnelle après un burnout. Références en Santé au Travail, 137, 59-71.

Borgogni, L., Consiglio, C., Alessandri, G., \& Schaufeli, W. B. (2012). "Do not throw the baby out with the bathwater!" Interpersonal strain at work and burnout. European Journal of Work and Organizational Psychology, 21(6), 875-898. doi: $10.1080 / 1359432 X .2011 .598653$

Byrne, B. M. (2010). Structural equation modeling with AMOS: Basic concepts, applications, and programming (2 ed.). New York: Routledge.

Carlotto, M. S., \& Câmara, S. G. (2004). Análise fatorial do Maslach Burnout Inventory (MBI) em uma amostra de professores de instituições particulares. Psicologia em Estudo, 9(3), 499-505. doi:10.1590/S1413-73722004000300018

Chou, L. P., Li, C. Y., \& Hu, S. C. (2014). Job stress and burnout in hospital employees: Comparisons of different medical professions in a regional hospital in Taiwan. BMJ Open, 4(2), 1-7. doi: 0.1136/bmjopen-2013-004185 
Cohen, A., Smith, M., \& Cohen, H. H. (1975). Safety program practices in high versus low accident rate companies: An interim report (questionnaire phase). National Institute for Occupational Safety and Health (p. 184). Washington, D.C.: Dept. of Health, Education, and Welfare, Public Health Service, Center for Disease Control. Recuperado de http://www.cdc.gov/ niosh/nioshtic-2/00048859.html

Consiglio, C. (2014). Interpersonal strain at work: A new burnout facet relevant for the health of hospital staff. Burnout Research, 1(2), 69 -75. doi:10.1016/j.burn.2014.07.002

De Andrade, A. L. (2014). Representações de grupos e percepção de risco no trabalho (Relatório Técnico de Pesquisa). Vitória: Fundação de Âmparo a Pesquisa do Espírito Santo.

Díaz, R. I., \& Cabrera, D. D. (1997). Safety climate and attitude as evaluation measures of organizational safety. Accident Analysis and Prevention, 29(5), 643-650. doi:10.1016/S0001-4575(97)00015-8

Fernandes, J. S., Miranzi, S. S. C., Iwamoto, H. H., Tavares, D. M. S., \& Santos, C. B. (2012). A relação dos aspectos profissionais na qualidade de vida dos enfermeiros das equipes Saúde da Família. Revista da Escola de Enfermagem da USP, 46(2), 404-412. doi:10.1590/S0080-62342012000200019

Ferreira, M. C., \& Mendes, A. M. B. (2008). Contexto de trabalho. In M. M. M. Siqueira (Ed.), Medidas do comportamento organizacional (pp. 111-123). Porto Alegre: Artmed.

Frasquilho, M. A. (2005). Medicina, uma jornada de 24 horas? Stress e burnout em médicos: Prevenção e tratamento. Revista Portuguesa de Saúde Pública, 23(2), 89-98.

Gershon, R. R., Karkashian, C. D., Grosch, J. W., Murphy, L. R., Escamilla-Cejudo, A., Flanagan, P. A., . . Martin, L. (2000). Hospital safety climate and its relationship with safe work practices and workplace exposure incidents. American Journal of Infection Control, 28(3), 211-221. doi:10.1067/mic.2000.105288

Gershon, R. R., Stone, P. W., Zeltser, M., Faucett, J., Macdavitt, K., \& Chou, S. S. (2007). Organizational climate and nurse health outcomes in the United States: A systematic review. Industrial Health, 45(5), 622-636. doi:10.2486/indhealth.45.622

Goldberg, D. P. (1972). The detection of psychiatric illness by questionnaire. Londres: Oxford University Press.

Gouveia, V. V., Chaves, S. S. S., Oliveira, I. C. P., Dias, M. R., Gouveia, R. S. V., \& Andrade, P. R. (2003). A utilização do QSG12 na população geral: Estudo de sua validade de construto. Psicologia: Teoria e Pesquisa, 19(3), 241-238. doi:10.1590/ S0102-37722003000300006

Hair, J. F., Anderson, R. E., Tatham, R. L., \& Black, W. C. (2005). Análise multivariada de dados. Porto Alegre: Bookman.

Halbesleben, J. R., Wakefield, B. J., Wakefield, D. S., \& Cooper, L. B. (2008). Nurse burnout and patient safety outcomes: Nurse safety perception versus reporting behavior. Western Journal of Nursing Research, 30(5), 560-577. doi:10.1177/0193945907311322

Heeb, J. L., \& Haberey-Knuessi, V. (2014). Health professionals facing burnout: What do we know about nursing managers? Nursing Research and Practice, 2014(2014), 1-7. doi:10.1155/2014/681814

Jasperse, M., Herst, P., \& Dungey, G. (2014). Evaluating stress, burnout and job satisfaction in New Zealand radiation oncology departments. European Journal of Cancer Care, 23(1), 82-88. doi:10.1111/ecc.12098

Kovaleski, D. F., \& Bressan, A. (2012). A síndrome de burnout em profissionais de saúde. Saúde \& Transformação Social, 3(2), 107-113.

Lautert, L. (1995). O desgaste profissional do enfermeiro (Tese de doutorado). Recuperado de http://www.lume.ufrgs.br/ handle/10183/11028

Madureira, N. (2012). Para maior satisfação no trabalho... apesar de ser na saúde. Goiás: Editora Futuro.

Maslach, C., \& Jackson, S. E. (1981). The measurement of experienced burnout. Journal of Organizational Behavior, 2(2), 99-113. doi:10.1002/job.4030020205

Minayo-Gomez, C., \& Thedim-Costa, S. M. F. (1997). A construção do campo da saúde do trabalhador: Percurso e dilemas. Cadernos de Saúde Pública, 13(Supl. 2), 21-32.

Montgomery, A., Panagopoulou, E., Kehoe, I., \& Valkanos, E. (2011). Connecting organisational culture and quality of care in the hospital: Is job burnout the missing link? Journal of Health Organization Management, 25(1), 108-123. doi:10.1108/14777261111116851

Montgomery, A., Todorova, I., Baban, A., \& Panagopoulou, E. (2013). Improving quality and safety in the hospital: The link between organizational culture, burnout, and quality of care. British Journal of Health Psychology, 18(3), 656-662. doi:10.1111/bjhp.12045 
Moreno, F. N., Gil, G. P., Haddad, M. C. L., \& Vannuchi, M. T. O. (2011). Estratégias e intervenções no enfrentamento da síndrome de burnout. Revista Enfermagem UERJ, 19(1), 140-145.

Murofuse, N. T., Abranches, S. S., \& Napoleão, A. A. (2005). Reflexões sobre estresse e burnout e a relação com a enfermagem. Revista Latino-Americana de Enfermagem, 13(2), 255-261. doi:10.1590/S0104-11692005000200019

Nicolau, M. T. (2014). O trabalho em unidades hospitalares: Particularidades profissionais e desafios institucionais. In M. R. Teixeira (Ed.), O multifacetado trabalho em unidades de saúde no século XXI (pp. 127-153). São Paulo: Dom Bosco.

Nogueira-Martins, L. A. (2003). A saúde do profissional de saúde. In M. A. De Marco (Ed.), A face humana da medicina: Do modelo biomédico ao modelo biopsicossocial (pp. 93-99). São Paulo: Casa do Psicólogo.

Pitta, A. (1990). Hospital: Dor e morte como ofício. Rio de Janeiro: Ed. Hucitec.

Profit, J., Sharek, P. J., Amspoker, A. B., Kowalkowski, M. A., Nisbet, C. C., Thomas, E. J., ... Sexton, J. B. (2014). Burnout in the NICU setting and its relation to safety culture. BMJ Quality and Safety, 23(10), 806-813. doi:10.1136/bmjqs-2014-002831

Puente-Palacios, K. E., \& Martins, M. D. C. (2013). Gestão do clima organizacional. In L. O. Borges \& L. Mourão (Eds.), O trabalho e as organizações: Atuações a partir da psicologia (Vol. 1, pp. 251-278). Porto Alegre: Artmed.

Richter, A., Kostova, P., Baur, X., \& Wegner, R. (2014). Less work: More burnout? A comparison of working conditions and the risk of burnout by German physicians before and after the implementation of the EU Working Time Directive. International Archives of Occupational and Environmental Health, 87(2), 205-215. doi:10.1007/s00420-013-0849-x

Sexton, J. B., Sharek, P. J., Thomas, E. J., Gould, J. B., Nisbet, C. C., Amspoker, A. B., Kowalkowski, M. A., Schwendimann, R., \& Profit, J. (2014). Exposure to Leadership WalkRounds in neonatal intensive care units is associated with a better patient safety culture and less caregiver burnout. BMJ Quality and Safety, 23(10), 814-822. doi:10.1136/bmjqs-2013-002042

Shimizu, H. E., \& Ciampone, M. H. T. (1999). Sofrimento e prazer no trabalho vivenciado pelas enfermeiras que trabalham em unidades de terapia intensiva em um hospital escola. Revista da Escola de Enfermagem da USP, 33(1), 95-106. doi:10.1590/ S0080-62341999000100010

Silva, J. L. L., \& Melo, E. C. P. (2006). Estresse e implicações para o trabalhador de enfermagem. Informe-se em Promoção da Saúde, 2(2), 16-18.

Slanetz, P. J. (2011). Ten steps to finding the right balance between career and family. American Journal of Roentgenology, 197(5), W814-W816. doi:10.2214/AJR.11.6481

Sprandel, L., \& Vaghetti, H. (2012). Valorização e motivação de enfermeiros na perspectiva da humanização do trabalho nos hospitais. Revista Eletrônica de Enfermagem, 14(4), 704-802. doi:10.5216/ree.v14i4.16100

Suñer-Soler, R., Grau-Martín, A., Flichtentrei, D., Prats, M., Braga, F., Font-Mayolas, S., \& Gras, M. E. (2014). The consequences of burnout syndrome among healthcare professionals in Spain and Spanish speaking Latin American countries. Burnout Research, 1(2), 82-89. doi:http://dx.doi.org/10.1016/j.burn.2014.07.004

Tamayo, M. R., \& Tróccoli, B. T. (2002). Exaustão emocional: Relações com a percepção de suporte organizacional e com as estratégias de coping no trabalho. Estudos de Psicologia (Natal), 7(1), 37-46. doi:10.1590/S1413-294X2002000100005

Tamayo, M. R., \& Tróccoli, B. T. (2009). Construção e validação fatorial da Escala de Caracterização do Burnout (ECB). Estudos de Psicologia (Natal), 14(3), 213-221. doi:10.1590/S1413-294X2009000300005

Thalhammer, M., \& Paulitsch, K. (2014). Burnout - Eine sinnvolle diagnose? Kritische überlegungen zu einem populären begriff. Neuropsychiatrie, 28(3), 151-159. doi:10.1007/s40211-014-0106-x

Trigo, T. R., Teng, C. T., \& Hallak, J. E. C. (2007). Síndrome de burnout ou estafa profissional e os transtornos psiquiátricos. Revista de Psiquiatria Clínica, 34(5), 223-233. doi:10.1590/S0101-60832007000500004

Wisetborisut, A., Angkurawaranon, C., Jiraporncharoen, W., Uaphanthasath, R., \& Wiwatanadate, P. (2014). Shift work and burnout among health care workers. Occupational Medicine (London), 64(4), 279-286. doi:10.1093/occmed/kqu009

Zohar, D. (1980). Safety climate in industrial organizations: Theoretical and applied implications. Journal of Applied Psychology, 65(1), 96-102. doi:10.1037/0021-9010.65.1.96 